\title{
Análise cinemática dos membros superiores em atletas de rugby em cadeira de rodas: estudo observacional
}

http://dx.doi.org/10.11606/1807-5509202000020283

\section{Resumo}

0 objetivo do presente estudo foi identificar o comportamento cinemático dos membros superiores (MMSS) de atletas de Rugby em Cadeira de Rodas (RCR) durante a tarefa de propulsão na cadeira de rodas. Neste estudo seccional foi realizada uma análise cinemática bidimensional dos MMSS de 19 atletas de RCR (idade $=31,5 \pm 5,6$ ), durante o teste de velocidade de 20 metros (VEL). Foram considerados os seguintes parâmetros: ângulos articulares, classe funcional e o questionário Whee/chair User's Shoulder Pain Index (WUSPI). A análise dos dados foi realizada com extração de valor de tendência central (média) e valor de dispersão (desvio padrão). As correlações foram verificadas pelo coeficiente de correlação de Spearman e Pearson. As variáveis cinemáticas analisadas estão descritas a seguir: ângulo de impulsão $\left(89,7^{\circ} \pm\right.$ $18,9)$, impulsão inicial $\left(31^{\circ} \pm 10,4\right)$, impulsão final $\left(66^{\circ} \pm 9,6\right)$, ângulo de contato $\left(97,1^{\circ} \pm 3,8\right)$, flexão de cotovelo $\left(94,2^{\circ} \pm 13,3\right)$, extensão de ombro $\left(66,6^{\circ} \pm 10,6\right)$, VEL $(7,7 s \pm 2,3)$ e ciclos de propulsão $(16,1$ $\pm 1,4$ ). Foi observado uma correlação significativa entre a Classificação Funcional (CF) e o VEL ( $r h o=$ $-0,70, p<0,05)$, assim como entre o tempo de prática de RCR com o VEL ( $r h o=0,54, p<0,05)$. No que diz respeito à identificação do grau de função dos MMSS, os atletas apresentaram boa funcionalidade com uma pontuação média no WUSPI de 17,8 \pm 8 pontos. Os atletas de RCR apresentaram um padrão cinemático com poucas variações espaço-temporais. Entretanto, foi possivel observar que alguns atletas tiveram melhor desempenho no teste de velocidade, principalmente os atletas com maior tempo de prática de RCR e com CF mais alta.

Palavras-chave: Cinemática; Cadeira de Rodas; Tetraplegia; Esportes para Pessoas com Deficiência.

\section{Introdução}

A análise do movimento humano no âmbito desportivo contribui de forma pujante para compreensão dos fenômenos do comportamento motor e, por conseguinte, sua influência sobre desempenho ${ }^{1-4}$. Essa premissa também tem sido estendida ao esporte adaptado, sobretudo em função do aumento do número e da qualidade técnica dos atletas nos últimos anos ${ }^{5-8}$. Dentre os esportes voltados para pessoas com deficiência física, destaca-se o Rugby em Cadeira de Rodas (RCR), que surgiu no Canadá, na década de $70^{9}$. Inicialmente, o RCR foi criado para atender indivíduos com lesões medulares altas e que, por isso, não eram elegíveis para a prática do basquetebol em cadeira de rodas. Assim, o RCR pode ser praticado por indivíduos com tetraplegia ou tetraequivalência, como lesão medular, sequela de poliomielite e amputaçōes, entre outros ${ }^{9,10}$.

O RCR combina basicamente elementos de 4 desportos, a saber: Rugby de sete, basquetebol, futebol americano e hockey no gelo. Durante o jogo, os atletas realizam tarefas como passes e arremessos, transição defesa-ataque, além de deslocamentos e troca de direção constante. Além disso, podem assumir posicionamentos distintos na equipe, atacante ou defensor, dependendo do grau de
*Centro Universitário
Augusto Motta, Rio de
Janeiro, RJ, Brasil.
${ }^{* *}$ Universidade Esta-
dual do Rio de Janeiro,
Rio de Janeiro, RJ,
Brasil.
***Universidade do
Estado Rio de Janeiro,
Rio de Janeiro, RJ,
Brasil.
***Departamento de
Fisioterapia, Universi-
dade Federal do Rio
de Janeiro, Rio de Ja-
neiro, RJ, Brasil. 
comprometimento motor ${ }^{6,10}$. Para que os indivíduos sejam incluídos nessa modalidade, é necessário que atendam à uma das categorias da classificação funcional (CF) proposta pela International Wheelchair Rugby Federation (IWRF), que varia entre 0,5 (indivíduos mais comprometidos) e 3,5 (indivíduos menos comprometidos) pontos, baseada na funcionalidade. No RCR, essa CF ainda é subdividida em atletas com ponto alto (2,0 $3,5)$ e baixo $(0,5-1,5)$. O somatório da CF dos quatro integrantes da equipe não deve ultrapassar o máximo de 8,0 pontos ou 8,5 quando a equipe for mista, ou seja, com atletas do gênero masculino e femino?.

Todas estas atividades executadas no RCR estão diretamente relacionadas ao status de uma boa função dos membros superiores (MMSS), principalmente no que diz respeito ao deslocamento da cadeira de rodas. Uma das tarefas mais executadas durante um jogo de RCR é a propulsão na cadeira de rodas, já que é um esporte com muita dinâmica de jogo. Desta forma, é imperativo a necessidade de uma boa funcionalidade dos MMSS e um bom entendimento dos aspectos biomecânicos relacionados a tarefa de propulsão no $\mathrm{RCR}^{8-10}$. Vale ressaltar, que apesar de a maioria dos usuários de cadeiras de rodas - esportistas ou não - relatarem dor no ombro, nem sempre os mesmos apresentam limitação funcional durante suas atividades diárias ${ }^{5,8}$. Entretanto, faz-se necessário a avaliação por meio de instrumentos confiáveis para identificar se o fator dor pode estar ou não causando impactos funcionais negativos, especialmente no que tange declínio no desempenho esportivo ${ }^{4,5}$.

Em virtude da sobrecarga imposta aos MMSS dos usuários de cadeira de rodas e as lesões relacionadas aos mesmos, os estudos de ZHAO et al. ${ }^{11}$, Russell et al. ${ }^{12}$ e Dellabiancia, Porcellini e Merolla ${ }^{13}$ descreveram o padrão cinemático dos MMSS, como por exemplo o movimento de propulsão. Por outro lado, no âmbito do RCR há uma escassez na literatura sobre as peculiaridades do comportamento motor dos praticantes, principalmente no que tange os aspectos biomecânicos. Aspectos como: tempo de prática da modalidade esportiva, cambagem da cadeira de rodas, CF dos atletas, tem demonstrado influência tanto na técnica de propulsão quanto na velocidade de deslocamento. Especialmente a CF, no qual os atletas com CF mais alta vem apresentando melhor desempenho nos teste motores, principalmente no que tange a velocidade de condução. Mas tais dados são escassos, sobretudo no que se refere a cinemática dos MMSS durante a tarefa de propulsão. Fato este, que torna esta tarefa um interessante objeto de estudo. Baseado neste contexto, o objetivo do presente trabalho foi analisar, descrever e correlacionar o comportamento cinemático e a função dos MMSS de um grupo de atletas do RCR durante a tarefa de propulsão na cadeira de rodas.

\section{Método}

\section{Delineamento do estudo e Amostra}

Neste estudo seccional foram avaliados 19 atletas de RCR, recrutados por conveniência no Rio de Janeiro/ Brasil. Todo processo de avaliação e análise dos dados foi feito por um avaliador único. A amostra foi composta por onze atletas pré-convocados para compor a Seleção Brasileira de RCR pré-Jogos Paralímpicos Rio 2016 (19 colocada no ranking da federação Internacional de Rugby em Cadeira de Rodas) e nove atletas da Equipe Santer Rio Rugby - Rio de Janeiro (6 ${ }^{\circ}$ colocada no ranking da liga nacional de RCR). Referente aos critérios de elegibilidade, foram incluídos atletas de RCR do sexo masculino, maiores de 18 anos de idade, com o tempo de treinamento de $\mathrm{RCR} \geq$ seis meses e que tivessem participado de pelo menos uma competição oficial no período anterior à pesquisa. Foram excluídos os participantes que apresentassem dor incapacitante para executar os movimentos relacionados a condução da cadeira de rodas no momento da avaliação e que estivessem ausentes dos treinamentos, em função de estarem sendo submetidos à tratamento ortopédico e/ou fisioterapêutico, além dos atletas que apresentassem amputaçōes de MMSS.

Este trabalho foi aprovado no comitê de ética em pesquisa do Centro Universitário Augusto MottaUNISUAM, sob aprovação número: 1.316.885, Rio de Janeiro - RJ, Brasil, e está de acordo com a Resolução 466/12 do Conselho Nacional de Saúde. Todos os participantes assinaram o Termo de 
Consentimento Livre e Esclarecido antes de serem incluídos no estudo.

\section{Protocolo}

Inicialmente, todos atletas responderam a uma anamnese para a aquisição de informações sociodemográficas e treinamento desportivo, a saber: idade, tempo de lesão, tempo de prática esportiva no RCR, tempo total de treino semanal, presença de dor no ombro e classificação funcional. Também foram feitas medidas da massa (Micheletti, Brasil) e comprimento corporal (em decúbito dorsal, foi feita medida da distância entre o plano do vértex e o plano da planta dos pés, com o auxílio de uma fita metálica não-flexível (Sanny; precisão $0,1 \mathrm{~cm})^{14}$.

Para identificar o grau de funcionalidade do ombro foi aplicado o questionário "Wheelchair Users Shoulder Pain Index” (WUSPI) na sua versão traduzida e adaptada para o português, para aqueles indivíduos com queixas álgicas no ombro identificados previamente. O WUSPI contém quinze itens e avalia o quanto a dor no ombro pode interferir em diferentes atividades diárias, como transferências da cadeira de rodas, autocuidado e propulsão. Cada item é pontuado de zero a dez, sendo que dez corresponde a dor no ombro que interfere completamente na atividade avaliada. Quanto maior a pontuação, maior a limitação funcional do indivíduo ${ }^{15,16}$.

\section{Análise cinemática}

A captura das imagens para análise cinemática (filmadora digital compacta Sony, modelo HDRSR10 HD, com frequência de 60 quadros por segundo, fabricada no Brasil) foi feita durante a propulsão na cadeira de rodas específica para a prática do RCR de cada atleta, no teste de velocidade de 20 metros (VEL), no local de treino das equipes.

Esse teste consiste em mensurar a habilidade de deslocamento da cadeira de rodas em velocidade, em um trajeto de 20 metros em linha reta ${ }^{17}$. A filmadora ficou fixada sobre o apoio de braço de uma cadeira de rodas auxiliar, independente, localizada ao lado direito dos atletas - a filmagem só foi realizada pelo lado direito. Isso permitiu a obtenção das imagens no plano sagital e análise de dados através de um sistema de coordenadas cartesianas. Durante a execução do VEL, um avaliador único acompanhou o deslocamento dos atletas, empurrando a cadeira de rodas - no qual a filmadora estava fixada - do início ao fim do percurso do teste.

\section{Delineamento do modelo biomecânico}

Os segmentos corporais foram identificados pela colocação de marcadores passivos esféricos de isopor com diâmetro de $25 \mathrm{~mm}$, na cor amarela, fixados em circunferências de PVC preto, com $40 \mathrm{~mm}$ de diâmetro. As variáveis cinemáticas analisadas foram: ângulo de impulsão, ângulo de contato, impulsão inicial e final, ângulo de flexão de cotovelo e extensão de ombro, ciclos de propulsão e velocidade final durante o VEL. Os marcadores foram colocados de acordo com um trabalho previamente realizado em usuários de cadeira de rodas não atletas ${ }^{18}$, cujos pontos de referência foram o ombro (acrômio), cotovelo (olécrano e epicôndilo lateral), punho (ponto central do punho, processo estilóide radial e ulnar) e eixo da roda.

\section{Variáveis espaço-temporais}

Os termos escolhidos para descrever os componentes temporais de deslocamento do membro superior durante a tarefa de propulsão foram: a) fase de impulsão inicial e; b) fase de impulsão final ${ }^{13}$. Os termos adotados para descrever os componentes espaciais que foram analisados (ângulos articulares ombro, cotovelo, ângulo de contato e impulsão) estão de acordo com a proposta de SLAVENS et al. ${ }^{18}$. Este, transcreve a partir de um sistema de coordenadas os ângulos articulares dos MMSS durante os dez primeiros ciclos da tarefa de propulsão na cadeira de rodas. As análises cinemáticas das variáveis de interesse foram realizadas por meio do software Kinovea (versão 8.23, São Paulo, Brasil).

\section{Análise estatística}

A análise estatística referente aos dados sociodemográficos e de caracterização da amostra, foi feita por meio da estatística descritiva com a extração de valor de tendência central (média) e valor de dispersão (desvio-padrão) para as variáveis numéricas contínuas e discretas e frequência relativa para as variáveis qualitativas.

O teste de Shapiro-Wilk foi aplicado para testar a distribuição das variáveis. Em seguida, para verificar as correlações entre as variáveis de interesse, foi usado o coeficiente de correlação de Spearman (rho) para os dados com distribuição não paramétrica e o 
Oliveira AS, et al.

coeficiente de correlação de Pearson ( $r$ ) para dados paramétricos, considerando valores (positivos ou negativos) com nível de significância de $\mathrm{p} \leq 0,05$ (SPSS 20.0 for Windows).

\section{Caracterização da Amostra}

A amostra foi composta por 19 atletas de RCR, com média de idade de 31,5 \pm 5,6 anos, massa corporal de 71,7 $\pm 8,9 \mathrm{~kg}$ e estatura de $177,9 \pm 6,9$ $\mathrm{cm}$, sendo todos os atletas com tetraequivalência decorrente de lesão medular. Cerca de 57,9\% dos atletas tinham CF entre 2,0 e 3,5 (ponto alto) e apresentavam tempo de prática de RCR superior a 60 meses $(65,6 \pm 29,6)$. No que diz respeito à identificação do grau de função dos MMSS, os atletas apresentaram uma pontuação média no questionário WUSPI de 17,8 \pm 8 pontos.

Os dados referentes à caracterização dos participantes do estudo estão apresentados na TABELA 1.

TABELA 1 -Características dos participantes do estudo sobre idade, tempo de lesão e classificação funcional. Valores apresentados em média, desvio-padrão e porcentagem.

RCR = Rugby em

Cadeira de Rodas; WUSPI $=$ Wheelchair Users Shoulder Pain Index.

\begin{tabular}{cc}
\hline & Atletas RCR ( $\mathbf{n}=\mathbf{1 9})$ \\
\hline Idade (anos) & $31,5 \pm 5,6$ \\
Massa corporal (kg) & $71,7 \pm 8,9$ \\
Estatura (cm) & $177,9 \pm 6,9$ \\
Tempo de lesão (anos) & $10,1 \pm 7,1$ \\
Tempo de Prática RCR (meses) & $65,6 \pm 29,6$ \\
Tempo total de treino semanal (horas) & $3,8 \pm 1,6$ \\
Classificação Funcional & Alta (57,9\%) \\
WUSPI & Baixa (42,1\%) \\
\hline
\end{tabular}

\section{Resultados}

Os valores médios e os respectivos desviospadrôes das variáveis ângulo de impulsão, impulsão inicial, impulsão final, ângulo de contato, abdução de ombro direito e esquerdo, flexão de cotovelo, extensão de ombro, VEL e ciclos de propulsão, estão apresentados na TABELA 2. Assim como os resultados da análise de correlação estáo apresentados na TABELA 3.

TABELA 2 -Resultados da análise cinemática do grupo de atletas de RCR.

\begin{tabular}{cc}
\hline & $\begin{array}{c}\text { Atletas RCR (n=19) } \\
\text { (Média } \pm \text { DP) }\end{array}$ \\
\hline Ângulo de impulsão (graus) & $89,7 \pm 18,9$ \\
Impulsão inicial (graus) & $31 \pm 10,4$ \\
Impulsão final (graus) & $66 \pm 9,6$ \\
Ângulo de contato (graus) & $97,1 \pm 3,8$ \\
Flexão de cotovelo (graus) & $94,2 \pm 13,3$ \\
Extensão de ombro (graus) & $66,6 \pm 10,6$ \\
VEL (segundos) & $7,7 \pm 2,3$ \\
Ciclos de propulsão (n ${ }^{\circ}$ de toques na cadeira) & $16,1 \pm 1,4$
\end{tabular}




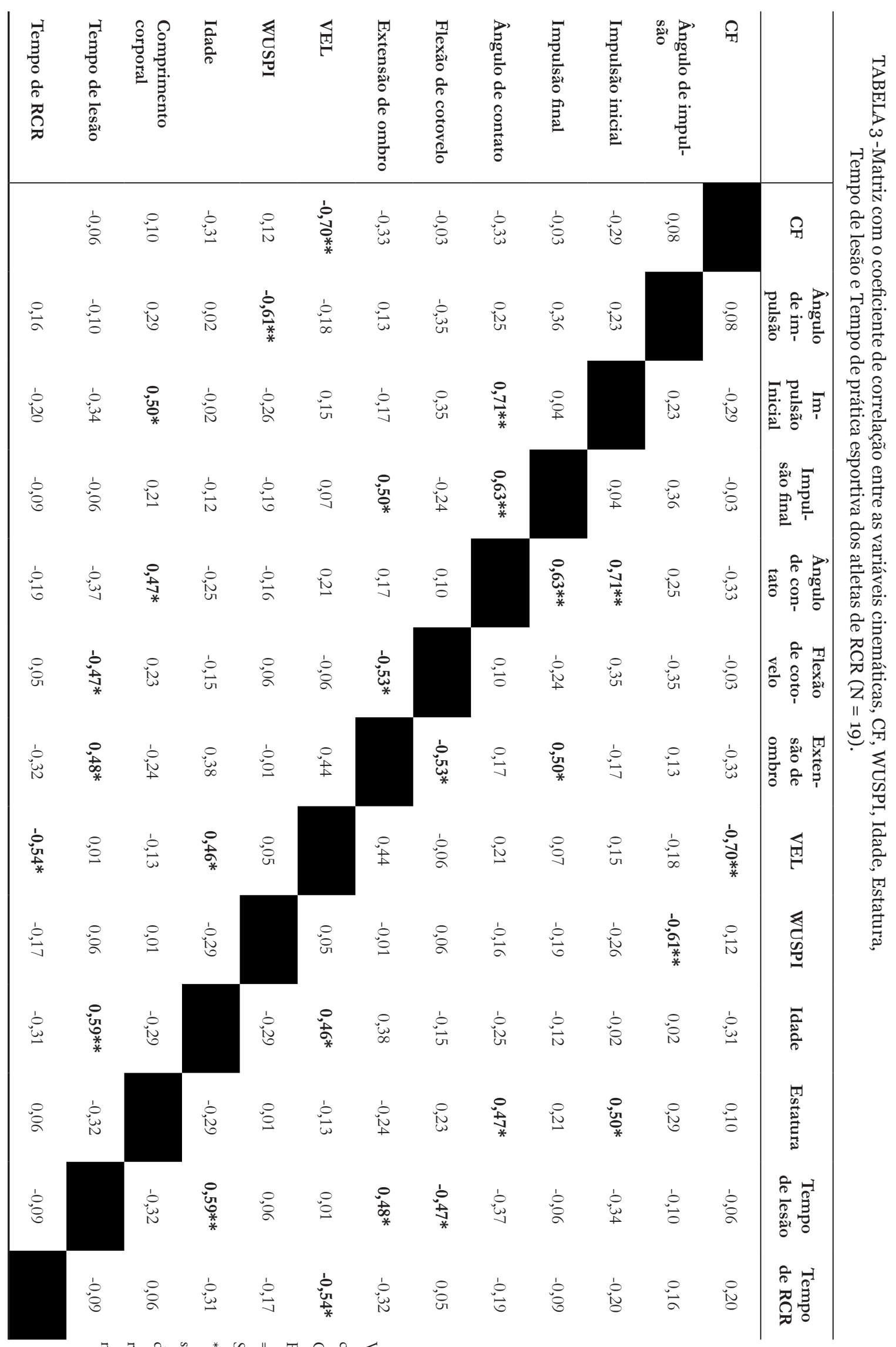




\section{Discussão}

O presente estudo investigou a cinemática dos MMSS durante a tarefa de propulsão na cadeira de rodas em atletas de RCR. Observamos, que os atletas apresentaram comportamento cinemático similar nas variáveis angulares durante a tarefa de propulsão na cadeira de rodas. Entretanto, foi possível identificar que os atletas com $\mathrm{CF}$ alta e maior tempo de prática de RCR tenderam a apresentar melhor desempenho durante o teste VEL, em virtude dos resultados encontrados durante as análises de correlação. No que tange a função do ombro, a pontuação média do questionário WUSPI foi baixa $(17,8 / 150)$, mostrando que a dor ombro não afeta de forma considerável as atividades diárias dos atletas. Vale ressaltar que as lesôes decorrentes do esporte não foram mensuradas.

\section{Avaliação funcional dos MMSS}

A dor no ombro é um achado comum em usuários de cadeira de rodas praticantes ou não de atividades esportivas ${ }^{16}$. A tarefa de propulsão na cadeira de rodas, destaca-se como um dos principais movimentos repetitivos responsáveis pela alta demanda imposta aos MMSS $8,15,16,19$. Portanto, é de fundamental importância se avaliar o impacto causado pela dor no ombro nas atividades diárias e esportivas, relacionadas a condução e manejo da cadeira de rodas. Dessa forma, é possível identificar o quanto esses indivíduos podem ficar limitados funcionalmente em decorrência da lesão ${ }^{20,21}$. Em nosso trabalho, a análise não apresentou correlação significativa $(p>0,05)$ entre a pontuação do WUSPI e o desempenho durante o teste de velocidade. Demonstrando que a pontuação do questionário não teve relação com o tempo final durante o VEL.

Entretanto, houve uma correlação negativa significativa $(p<0,01)$ entre o WUSPI e a variável ângulo de impulsão. Uma das hipóteses para esse resultado, é que os atletas com menor pontuação no WUSPI e consequentemente menor limitação funcional, tendem a ter uma maior capacidade em aumentar o ângulo de impulsão.

O questionário WUSPI é amplamente usado para avaliar a dor e função dos MMSS em usuários de cadeira de rodas, mostrando-se um instrumento confiável para tal finalidade ${ }^{15,20,22-25}$. Entretanto, a maioria dos trabalhos são relacionados a usuários de cadeira de rodas não praticantes de atividades esportivas $^{15,20,22}$.

Dentro desse contexto, nossos resultados demonstraram que os atletas que relataram dor no ombro não apresentaram uma limitação funcional severa, sugerindo que, possivelmente, o questionário WUSPI pode não ser considerado um instrumento sensível para atletas usuários de cadeira de rodas. Tal fato pode ser atribuído à ausência de perguntas relacionadas a dor e atividade esportiva no questionário, já que o WUSPI contém apenas 1 item $(1-15)$ relacionado a pratica esportiva. Vale ressaltar, que a dor é um achado inerente ao esporte de alto rendimento, inclusive no paradesporto ${ }^{8}$. Em virtude disso, os atletas podem estar acostumados a conviver com a dor, de forma que a mesma acaba não causando um impacto considerável em suas atividades básicas diárias.

\section{Análise cinemática}

Vários fatores podem influenciar na velocidade de deslocamento dos usuários de cadeira de rodas, como o tempo de utilização da cadeira de rodas ${ }^{26}$, tipo de superfície ${ }^{22}$, estabilidade do tronco e função dos MMSS $^{7}$, nível de classificação funcional ${ }^{19,26}$, além de aspectos biomecânicos, como os ângulos de impulsão e contato ${ }^{27,28}$.

O ângulo de contato na cadeira de rodas corresponde a fase em que as mãos estão em contato direto com o aro, iniciando-se na impulsão inicial, até a liberação do contato das mãos com o aro na fase de impulsão final ${ }^{13}$. No presente trabalho, nossos resultados são conflitantes com de outros autores ${ }^{13,27,28}$, que sugerem que o aumento do ângulo de impulsão e ângulo de contato podem favorecer a um aumento da velocidade durante o deslocamento da cadeira de rodas.

$\mathrm{Na}$ análise, nossos resultados não apontaram relação significativa entre as variáveis VEL, ângulo de contato e ângulo de impulsão. Ou seja, essas variáveis, teoricamente, não influenciaram na velocidade final durante o teste. Entretanto, foi possível observar uma correlação significativa positiva entre o ângulo de contato, impulsão inicial e impulsão final, sugerindo que o aumento do ângulo de impulsão inicial e final influencia em um maior ângulo de contato. Resultado este esperado, pois o ângulo de contato é definido pela 
soma do ângulo de impulsão inicial com o ângulo de impulsão final.

Nesse sentido, vale ressaltar que nenhuma das variáveis angulares apresentou correlação significativa com o teste VEL, incluindo o ângulo de contato e ângulo de impulsão. Estes, são citados como ângulos que podem influenciar em uma maior velocidade de deslocamento da cadeira de $\operatorname{rodas}^{13,27}$, fato que não foi observado no nosso trabalho. Dessa forma, os resultados sugerem que o desempenho durante o teste VEL não foi influenciado pelas variáveis cinemáticas.

Entretanto, o teste VEL apresentou uma correlação negativa significativa com o tempo de prática de RCR ( $r h o=-0,54, \mathrm{p}<0,05$ ). Em virtude disso, é possível sugerir que o tempo de prática de RCR pode influenciar no tempo final durante o teste de velocidade. Sendo os atletas que apresentam maior tempo de prática, tendendo a ter um melhor desempenho. De certa forma, esses dados vão de acordo com outros autores ${ }^{26}$, que observaram que o tempo de utilização da cadeira de rodas pode influenciar na velocidade de deslocamento da mesma. Os autores sugerem que quanto maior o tempo de relação entre o usuário e a cadeira de rodas, melhor é o desempenho motor durante a tarefa de propulsão, baseandose principalmente no princípio de aprendizagem motora.

Nesse contexto, como visto em nossos resultados, os atletas que praticam a mais tempo o RCR, possivelmente por terem mais familiaridade com as tarefas sobre a cadeira de jogo (processo de aprendizagem motora), sobretudo o ato de propulsão, tiveram um melhor desempenho durante o teste de velocidade. Ainda na correlação intragrupo, foi possível observar que os atletas apresentaram uma relação negativa significativa entre a flexão de cotovelo e extensão de ombro $(r h o=-0,53, \mathrm{p}<0,05)$. Sugerindo que a medida que o ângulo de extensão do ombro aumenta, o ângulo de flexão do cotovelo diminui.

Segundo Dellabiancia et al. ${ }^{13}$, durante a fase de impulsão na cadeira de rodas, as mãos realizam um movimento semicircular, que transmite uma força resultante para cima quando o cotovelo está fletido e com ombro em extensão (entre o início e o meio do impulso). Posteriormente, ocorre uma força para baixo, quando acontece a extensão do cotovelo (do meio para o fim do movimento). Ocorrendo assim, uma grande carga atuante na articulação do ombro.
Dessa forma, a relação significativa entre os ângulos de flexão de cotovelo e extensão de ombro, pode ser uma estratégia motora usada pelos atletas para favorecer o deslocamento da cadeira de rodas. Isto, devido a um provável aumento da força resultante gerada pelos MMSS durante o deslocamento da cadeira de rodas. Levando em consideração que os atletas de RCR, na maioria das vezes, são pessoas com lesão medular que apresentam comprometimento da força muscular dos MMSS $^{9,10}$, essa estratégia pode ser de fundamental importância para auxiliar no deslocamento da cadeira de jogo de forma mais efetiva.

\section{Propulsão na cadeira de rodas: atletas de RCR, outros esportes sobre cadeira de rodas e usuários de cadeira de rodas não atletas}

O movimento de propulsão na cadeira de rodas é amplamente estudado ${ }^{13,18-20}$. Porém, a maioria dos estudos foram desenvolvidos em usuários de cadeira de rodas não atletas e paraplégicos ${ }^{4,11-13,18-22}$, o que dificulta a comparação com os resultados do nosso trabalho.

No estudo realizado por Soltau et al. ${ }^{28}$, os autores se propuseram a avaliar a simetria dos MMSS em sujeitos com paraplegia $(n=80)$, durante a tarefa de propulsão na cadeira de rodas em diferentes velocidades, por meio de uma análise cinemática e cinética. Os resultados apontaram que não houve assimetria significativa nos dados cinéticos e cinemáticos, com exceção do ângulo de impulsão. Nesse estudo, o ângulo de impulsão médio registrado durante as diferentes velocidades foi de $79,6^{\circ}$. Este valor, tem cerca de $10^{\circ}$ a menos do que a média encontrada em nossos resultados no grupo de atletas de $\operatorname{RCR}\left(89,7^{\circ} \pm 18,9^{\circ}\right)$.

Dentro desse contexto, é possível considerar que os atletas de RCR apresentam comportamentos cinemáticos distintos no que tange o ângulo de impulsão. Vale ressaltar que a amostra do estudo de SOLTAU et al. ${ }^{28}$ foi composta por usuários de cadeira de rodas não atletas e paraplégicos, o que dificulta a comparação dos resultados com os nossos.

Porém, é possível que os atletas de RCR apresentem um maior ângulo de impulsão do que os usuários de cadeira de rodas paraplégicos, devido a força muscular debilitada dos MMSS, usando essa estratégia para aumentar a velocidade de deslocamento da cadeira de rodas. Haja visto que, alguns autores ${ }^{13,26,27}$ sugerem que o 
aumento do ângulo de impulsão pode favorecer a um aumento da velocidade durante a tarefa de propulsão na cadeira de rodas.

A compreensão dos fenômenos cinéticos e cinemáticos acerca da cadeira de rodas, tem importância fundamental para melhorar a interação usuário-cadeira ${ }^{4,13}$. Tal compreensão tem auxiliado no desenvolvimento das modalidades paradesportivas, como no basquete em cadeira de rodas $^{7,29}$ e no $\mathrm{RCR}^{6}$. Contudo, ainda existe um vácuo literário acerca dessa temática, sobretudo no RCR. Fato este, que pode ser atribuído - não exclusivamente - a falta de incentivo ao esporte adaptado, principalmente quando comparado aos esportes convencionais.

Nesse sentido, com o intuito de colaborar para uma melhor compreensão do comportamento motor dos atletas usuários de cadeira de rodas, Crespo-Ruiz, Ama-Espinosa e Gil-Agudo ${ }^{29}$ realizaram um trabalho para analisar a cinemática dos membros superiores em atletas de basquete em cadeira de rodas durante a tarefa de propulsão, considerando a classificação funcional de cada atleta. Os autores observaram que o ângulo de contato parece reduzir com o aumento da classificação funcional. Em contrapartida a isso, apesar de nossos resultados apresentarem uma tendência de correlação negativa entre o ângulo de contato e $\mathrm{CF}$, tal relação não foi significativa (rho $=-0,33, \mathrm{p}>0,05)$. Entretanto, no trabalho de Crespo-Ruiz, Ama-Espinosa e Gil-Agudo ${ }^{29}$, os autores não realizaram nenhuma estatística de correlação. Sugerindo que o aumento do ângulo de contato apresentou uma relação inversa a $\mathrm{CF}$, apenas pelos dados encontrados na estatística descritiva.

Contudo, mais uma vez é importante ressaltar diferenças importantes nos estudos. No trabalho supracitado $^{29}$, a amostra foi composta por atletas de basquete em cadeira de rodas e, sabendose das peculiaridades de cada modalidade, o comportamento cinemático entre os atletas de RCR e basquete em cadeira de rodas pode variar consideravelmente.

Devido à escassez de trabalhos relacionados ao comportamento cinemático dos atletas de RCR, só foi encontrado um trabalho que abordasse essa temática. Neste, SARro et al. ${ }^{6}$ analisaram a dinâmica do movimento de jogadores de elite de RCR durante um jogo de alto nível. Os resultados apontaram que os jogadores percorreram maior distância e tiveram maior velocidade média no primeiro tempo de jogo. Sendo as menores velocidades notadas mais frequentemente em jogadores com menor capacidade funcional. Tais achados também foram observados em nossos resultados, demonstrando que atletas com menor CF tendem a ter um desempenho inferior durante tarefas motoras. Nesse trabalho ${ }^{6}$, os autores analisaram somente o comportamento cinemático linear. Não foram observados aspectos angulares, como: ângulos articulares, ângulos de contato e impulsão. Fatos que reforçam a importância do nosso trabalho para a compreensão do RCR.

Dentro desse contexto, os resultados do presente trabalho apontam que os atletas de RCR apresentam comportamentos cinemáticos similares na maioria das variáveis estudadas, a saber: flexão de cotovelo, extensão de ombro, impulsão inicial e final, ângulo de contato, ângulo de impulsão e ciclos de propulsão. Entretanto, nas variáveis espaço-temporais foi possível observar um comportamento distinto no desempenho durante o teste de velocidade de 20 metros. Vale ressaltar, que a análise cinemática no presente estudo foi realizada no local de treino dos atletas, com as cadeiras de jogo de cada atleta. Fato importante, pois a maioria dos estudos relacionados ao movimento de propulsão na cadeira de rodas ${ }^{4,8,12,13,18,20-22,27,28,30}$, são realizados em laboratório com esteiras estacionárias.

Uma limitação a ser considerada no presente estudo, é o fato de não termos analisado o padrão da mão adotado pelos atletas durante a propulsão na cadeira de rodas (arco, laço único, laço duplo e semicircular) ${ }^{13,17}$. Pois existem dados recentes ${ }^{31}$ apontando que diferentes tipos de padrão podem impor demandas distintas aos MMSS, podendo influenciar no deslocamento da cadeira de rodas. Este ponto é particularmente relevante, tratandose de atletas com tetraplegia, que normalmente apresentam pouca funcionalidade das mãos. Para trabalhos futuros sugere-se incluir a análise do padrão da mão.

Apesar do crescimento das modalidades paradesportivas em todo mundo e o aumento das pesquisas acerca desse tema ${ }^{5-10,16,17}$, um fator que ainda dificulta no desenvolvimento de trabalhos científicos é a dificuldade de conseguir amostras com tamanhos representativos. Tal dificuldade atribui-se, em parte, ao baixo número de praticantes de atividades paradesportivas.

No RCR, a principal dificuldade é decorrente 
do baixo número de tetraplégicos na população em geral ${ }^{26}$, fato que dificulta ainda mais a captação de pessoas indicadas para esta modalidade. O baixo número de participantes em alguns estudos, acaba limitando a extrapolação dos resultados encontrados para a população em geral do esporte estudado. No nosso trabalho, o $\mathrm{N}$ amostral foi composto por 19 atletas de RCR, um número acima do encontrado em alguns trabalhos recentes sobre $\mathrm{RCR}^{6,10,16,17,19}$. Vale ressaltar, que mesmo com diversos estudos sobre o movimento de propulsão na cadeira de rodas em atletas ou não-7,12,13,18-22, no RCR esse tema ainda permanece escasso. Fato este, que contribuiu para elaboração e execução do presente trabalho.

Em conclusão, os atletas de RCR apresentaram comportamento cinemático similar na maioria das variáveis cinemáticas estudadas, apresentando um padrão com poucas variações. Sugerindo assim, que apesar das diferentes classes funcionais, o padrão cinemático dos atletas mostrou-se bem similar. Entretanto, foi possível observar que alguns atletas tiveram melhor desempenho no teste de velocidade, principalmente os atletas com maior tempo de prática de RCR. Nossos resultados ajudam a fornecer novas informaçôes sobre o comportamento motor dos atletas de RCR - haja visto que existem poucos trabalhos acerca dessa temática - e podem ajudar treinadores, clínicos e pesquisadores a compreender melhor os fenômenos cinemáticos acerca dessa modalidade ainda pouco explorada.

\title{
Conflito de interesses
}

Os autores declaram não haver conflitos de interesses.

\section{Agradecimentos}

Agradecemos aos membros e voluntários do programa de pós-graduação stricto-sensu do Centro Universitário AugustoMotta (UNISUAM) - RJ.

\begin{abstract}
Kinematic analysis of upper limbs in wheelchair rugby athletes: observational study

The aim of this study was to identify kinematic behavior of the upper limbs during task wheelchair propulsion. In this cross-sectional study, we performed a two-dimensional kinematic analysis of the upper limbs of 19 Wheelchair Rugby (WR) athletes during the 20-meter velocity test. Data analysis was performed by descriptive statistics, with central tendency (mean) and dispersion (standard deviation). Correlations were verified by Spearman's correlation coefficient and Pearson's correlation coefficient. SPSS 20.0 software for Windows was used for all analyses. Kinematic analyses were performed in Kinovea 8.23 software. Data kinematic variables during wheelchair propulsion task are described below: impulse angle $\left(89.7^{\circ} \pm 18.9\right)$, initial impulse $\left(31^{\circ} \pm 10.4\right)$, final impulse $\left(66^{\circ} \pm 9.6\right)$, contact angle $\left(97.1^{\circ} \pm 3.8\right)$, elbow flexion $\left(94.2^{\circ} \pm\right.$ $13.3)$, shoulder extension $\left(66.6^{\circ} \pm 10.6\right)$, speed test $\left(7.7^{\circ} \pm 2,3\right)$ and propulsion cycles $\left(16.1^{\circ} \pm 1.4\right)$. We observed significant correlation between Functional Classification (FC) velocity test ( $r$ o $=-0.70, p<0.05$ ) and between WR practice time with velocity test $(r h o=0.54, P<0.05)$. In the identification of degree function upper limbs, there is a WUSPI questionnaire of $17.8 \pm 8$ points. In conclusion, WR athletes presented similar kinematic behaviour in most variables, presenting a pattern with few variations. However, some athletes had better performance in velocity test, especially athletes with longer WR practice time and higher FC.
\end{abstract}

KeYwords: Biomechanical Phenomena; Wheelchair; Quadriplegia; Sports for Persons with Disabilities. 


\section{Referências}

1. Stefanyshyn DJ, Stergiou P, Lun VM, Meeuwisse WH, Worobets JT. Knee angular impulse as predictor of patellofemoral pain in runners. Am J Sports Med. 2006;34(11):1844-51.

2. Hirata R, Duarte M. Efeito da posição relativa do joelho sobre a carga mecânica interna durante o agachamento. Rev Bras Fisioter. 2007;11(2):121-5.

3. Miana AN, Schor B, Castropil W, Bitar AC, Duarte M. Discinesia escapular: avaliação clínica e análise cinemática tridimensional. RBM Ver Bras Med. 2009;66(suppl1).

4. Gagnon G, Babineau A, Champagne A, Desroches G, Aissaoui R. Trunk and Shoulder Kinematic and Kinetic and Electromyographic Adaptations to Slope Increase during Motorized Treadmill Propulsion among Manual Wheelchair Users with a Spinal Cord Injury. Biomed Res Int. 2015;2015:636319.

5. Reid M, Elliott B, Alderson J. Shoulder joint kinetics of the elite wheelchair tennis serve. Br J Sports Med. 2007;41:739_ 744.

6. Sarro KJ, Misuta MS, Burkett B, Malone LA, Barros RM. Tracking of wheelchair rugby players in the 2008 Demolition Derby final. J Sports Sci. 2010;28(2):1-8.

7. Yanci J, Granados C, Otero M, Badiola A, Olasagasti J, Bidaurrazaga-Letona I, et al. Sprint, agility, strength and endurance capacity in wheelchair basketball players. Biology of Sport. 2015;32(1).

8. Aytar A, Zeybek A, Pekyavas ON, Tigli AA, Ergun N. Scapular resting position, shoulder pain and function in disabled athletes. Prosthet Orthot Int. 2015;39(5):390-6.

9. IWRF - INTERNATIONAL WHEELCHAIR RUGBY FEDERATION. IWRF CLASSIFICATION MANUAL. 3a ed. Delta: IWRF;2015 [citado 04 jul 2016]. Disponível em: http://www.iwrf.com/resources/iwrf_docs/IWRF_Classification_Manual_3rd_Edition_rev-2015_(English).pdf.

10. Campana BM, Gorla IJ, Duarte E, Scaglia JA, Tavares FCGCM, Barros FJ. O Rugby em Cadeira de Rodas: aspectos técnicos e táticos e diretrizes para seu desenvolvimento. Motriz. 2011;17(4):748-757.

11. Zhao KD, Van Straaten MG, Cloud BA, Morrow MM, An K-N, Ludewig PM. Scapulothoracic and Glenohumeral Kinematics During Daily Tasks in Users of Manual Wheelchairs. Frontiers in Bioengineering and Biotechnology. 2015;3:183. doi:10.3389/fbioe.2015.00183.

12. Russell IM, Raina S, Requejo PS, Wilcox RR, Mulroy S, McNittGray JL. Modifications in wheelchair propulsion technique with speed. Front Bioeng Biotechnol. 2015;3:171.

13. Dellabiancia F, Porcellini G, Merolla G. Instruments and techniques for the analysis of wheelchair propulsion and upper extremity involvement in patients with spinal cord injuries: current concept review. Muscles Ligaments Tendons J. 2013;3(3):150-156.

14. Guedes DP, Guedes JERP. Manual Prático para Avaliação em Educação Física. São Paulo: Manole; 2006.

15. Curtis KA, Roach KE, Applegate EB, Amar T, Benbow CSTD, Genecco TD, et al. Reliability and validity of the Wheelchair User's Shoulder Pain Index (WUSPI). Paraplegia. 1995;33:595-60.

16. Pereira MM, Gorla JI. Avaliação da intensidade da dor no ombro em atletas com deficiência física praticantes de modalidades esportivas coletivas. Arq Ciênc Saúde UNIPAR. 2010;14(3):231-235.

17. Gorla JI, Costa e Silva AA, Costa LT, Campos LFCC. Validação da bateria "Beck" de testes de habilidades para atletas brasileiros de "rugby" em cadeira de rodas. Rev Bras Educ Fís Esporte. 2011;25(3):473-86.

18. Slavens BA, Schnorenberg AJ, Aurit CM, Graf A, Krzak JJ, Reiners K, et al. Evaluation of Pediatric Manual Wheelchair Mobility Using Advanced Biomechanical Methods. Biomed Res Int. 2015;2015:634768

19. Bussmann AJC, Greguol M. Análise das técnicas de propulsão de atletas praticantes de modalidades em cadeira de rodas: um estudo de revisão. Rev Ter Ocup Univ São Paulo. 2012;23(2):193-198.

20. Requejo PS, Mulroy SJ, Ruparel P, Hatchett PE, Haubert LL, Eberly VJ, et al. Relationship Between Hand Contact Angle and Shoulder Loading During Manual Wheelchair Propulsion by Individuals with Paraplegia. Top Spinal Cord Inj Rehabil. 2015;21(4):313-24.

21. Rice IM, Jayaraman C, Hsiao-Wecksler ET, Sosnoff JJ. Relationship between shoulder pain and kinetic and temporal-spatial variability in wheelchair users. Arch Phys Med Rehabil. 2014;95(4):699-704.

22. Cowan RE, Nash MS, Collinger JL, Koontz AM, Boninger ML. Impact of Surface Type, Wheelchair Weight, and Axle Position on Wheelchair Propulsion by Novice Older Adults. Arch Phys Med Rehabil. 2009;90(7):1076-1083.

23. Fagher K, Lexell J. Sports-related injuries in athletes with disabilities. Scand J Med Sci Sports. 2014;24:e320-e331. doi: $10.1111 /$ sms. 12175 . 
24. Van Straaten M, Cloud BA, Morrow MM, Ludewig PM, Zhao KD. Effectiveness of Home Exercise on Pain, Function, and Strength of Manual Wheelchair Users With Spinal Cord Injury: A High-Dose Shoulder Program With Telerehabilitation. Arch Phys Med Rehabil. 2014;95(10):1810-1817.e2.

25. Bauerfeind J, Koper M, Wieczorek J, Urbański P, Tasiemski T. Sports Injuries in Wheelchair Rugby - A Pilot Study. J Hum Kinet. 2015;48:123-132. doi:10.1515/hukin-2015-0098.

26. Requejo PS, Jan Furumasu BS, Mulroy SJ. Evidence-Based Strategies for Preserving Mobility for Elderly and Aging Manual Wheelchair Users. Top Geriatr Rehabil. 2015;31(1):26-41.

27. Requejo OS, McNitt-Gray JL. Editorial: Wheelchair Mobility Biomechanics. Front Bioeng Biotechnol. 2016;4:53.

28. Soltau SL, Slowik JS, Requejo OS, Mulroy SJ, Neptune RR. An Investigation of Bilateral Symmetry During Manual Wheelchair Propulsion. Front Bioeng Biotechnol. 2015;3:86.

29. Crespo-Ruiz BM, Ama-Espinosa AJD, Gil-Agudo AM. Relation Between Kinematic Analysis of Wheelchair Propulsion and Wheelchair Functional Basketball Classification. Adapt Phys Activ Q. 2011;28:157-172.

30. Cowan RE, Nash MS, Collinger JLBS, Koontz AM, Boninger ML. Impact of Surface Type, Wheelchair Weight, and Axle Position on Wheelchair Propulsion by Novice Older Adults. Arch Phys Med Rehabil. 2009;90(7):1076-83.

31. Slowik JS, Requejo PS, Mulroy SJ, Neptune RR. The influence of wheelchair propulsion hand pattern on upper extremity muscle power and stress. J Biomech. 2016;49(9):1554-61.

ENDEREÇO DE CORRESPONDÊNCIA: Júlio Guilherme Silva Av. Paris, $\mathrm{n}^{\mathrm{o}} 34-3^{\mathrm{o}}$ andar - Bonsucesso Rio de Janeiro - RJ - BRASIL CEP: 21041-020 E-mail:jglsilva@yahoo.com.br
Submetido: 26/06/2017

$1^{\text {a }}$ revisão: $26 / 10 / 2017$

$2^{\mathrm{a}}$ revisão: $11 / 04 / 2018$

Aceito: 30/05/2018 